\title{
Analysis of the wall shear stress in a generic aneurysm under pulsating and transitional flow conditions
}

\author{
Andreas Bauer ${ }^{1}$ (1) $\cdot$ Maximilian Bopp ${ }^{1} \cdot$ Suad Jakirlic ${ }^{1}$ Cameron Tropea ${ }^{1} \cdot{\text { Axel Joachim } \mathrm{Krafft}^{1} \cdot \text { Nina Shokina }}^{1}$. \\ Jürgen Hennig ${ }^{1}$
}

Received: 23 October 2019 / Revised: 9 January 2020 / Accepted: 25 January 2020 / Published online: 6 February 2020

(c) The Author(s) 2020

\begin{abstract}
In this study, the flow through a generic abdominal aneurysm under realistic pulsating flow conditions is examined with magnetic resonance velocimetry (MRV), laser Doppler velocimetry (LDV) and computational fluid dynamics (CFD). The influence of flow phenomena on the wall shear stress (WSS) is examined. It is seen that a strong vortex ring develops during systole at the proximal end of the aneurysm and subsequently travels downstream and decays. The vortex formation plays a major role in the temporal and spatial distribution of the WSS, which is analyzed in detail. A peak of the WSS is observed for a very limited time and in a very localized region where the vortex ring initially develops. The intrinsic temporal averaging during the acquisition of the MRV data is found to significantly decrease this peak. CFD and LDV results, which are averaged in the same manner, show a similar behavior. This indicates that besides the spatial resolution, the temporal resolution is a crucial factor, which needs to be considered especially in flows where vortex rings are observed. Results from LDV and CFD show excellent agreement for the velocity field obtained by MRV. While the flow is found to be laminar in the undilated diameter, results show laminar-turbulent transitional behavior for specific phases of the cycle within the aneurysm bulk. Although MRV is not capable of measuring instantaneous velocity fluctuations, we show that the periodic increase in turbulence intensity can be observed from image artifacts in the MRV data. These artifacts increase the velocity uncertainty, which correlates well with the velocity fluctuations measured with LDV. Although the flow encounters laminar and transitional conditions as well as multiple vortices and stagnation and reattachment points, the improved instabilitysensitive Reynolds stress model, which is used for the numerical simulations of this work, shows very good agreement with the measurements. Significant effort has been expended by numerous research groups in recent years in improving the estimation of WSS from MRV data. However, an assessment of these various post-processing methods is only possible if the true values of the WSS are known. The present study is therefore aimed at providing such ground truth WSS values as well as the corresponding MRV data, allowing also other research groups to validate their WSS estimation methods using the experimental data set presented in this work.
\end{abstract}

\section{Graphic Abstract}
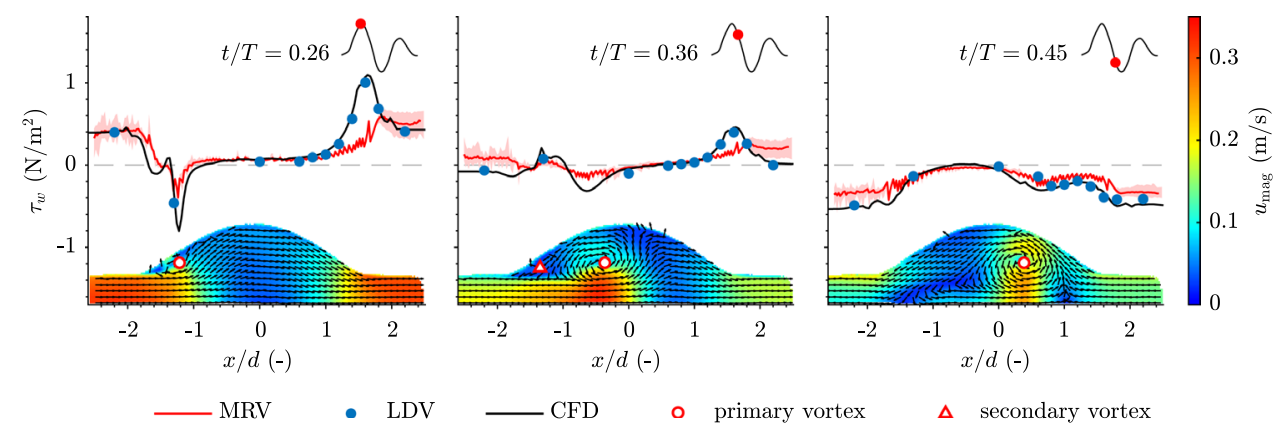

$\Delta \quad$ secondary vortex

Cameron Tropea

tropea@sla.tu-darmstadt.de

Extended author information available on the last page of the article 


\section{Introduction}

In cardiovascular medicine, abnormal changes of the vessel geometry are of great interest. A prominent example of such a change is the enlargement of the aortic vessel diameter, so-called abdominal aortic aneurysms (Aggarwal et al. 2011), whose initiation and growth triggers are subject to current discussion (Kemmerling and Peattie 2018). In general, the question needs to be answered, under which conditions the rupture of such an aneurysm is most likely (Fillinger et al. 2002; Munarriz et al. 2016), and at which stage of development surgical intervention is reasonable?

There exist different biomarkers and biochemical processes, which influence the vessel walls (Lasheras 2007). Almost all investigations agree that the wall shear stress (WSS) is one of these and of major importance in the emergence of such cardiovascular diseases (van Ooij et al. 2017). However, different opinions exist about which quantitative values of the wall shear stress initiate which kind of disease. Since the WSS is highly temporally and spatially distributed, as well as being a vectorial quantity, the specification of a single value may not be appropriate. The general assumption at the moment is that very high or low magnitudes of the WSS contribute to aneurysm growth (Boussel et al. 2008; Watton et al. 2011; Miura et al. 2013; Boyd et al. 2016), while spatial gradients provoke the initial development (Boussel et al. 2008; Meng et al. 2007; Dolan et al. 2013). However, no consensus exists on these issues and the discussion is still in progress. Arzani and Shadden (2016) provide a good overview of the current state of the art. The overall uncertainty arises furthermore from the fact that there is currently no reliable and overall accepted method to accurately measure the WSS in vivo. The most common practice is a measurement with phase contrast magnetic resonance imaging (PC-MRI), also termed magnetic resonance velocimetry (MRV), which has gained much attention in the last few years due to the work of Markl et al. (2012). Although MRV measurements are commonly used to measure the WSS, they may be subjected to large measurement errors and uncertainties, caused by the limited spatial resolution.

Current progress in measurement of the aneurysm WSS distribution follows one of two possible approaches: On the one hand, the overall wall shear stress estimation from MRV data itself can be improved. On the other hand, there exist several experimental in vitro measurements and computational fluid dynamics (CFD), which focus on the general understanding of aneurysm flow. By gaining a deeper understanding of the flow phenomena, WSS results may be better interpreted and measurement parameters can be better chosen.

The modeling of the aneurysm geometry can be divided into the analysis of patient-specific and generic models.
The advantage of the former is that specific information, e.g., before surgical intervention, can be obtained, allowing an individual prediction of the existing in vivo flow field. However, generic models provide a more general description of the problem, allowing a better comparison between different research groups, different WSS estimators and CFD codes. Exactly for this latter reason, we have used a generic geometry of an abdominal aortic aneurysm in the present study.

There exist numerous experimental investigations of aneurysms. Budwig et al. (1993) and Asbury et al. (1995) were the first who experimentally investigated the flow through a symmetric generic aneurysm and obtained preliminary results for the spatial distribution of the wall shear stress. They concluded that in the areas around the attachment and reattachment points, strong spatial gradients and high WSS peaks exist. However, both studies examined only steady flow conditions. Further experimental investigations were later conducted by Egelhoff et al. (1999) and Deplano et al. (2007, 2013, 2016), who considered pulsating flow rates. Deplano analyzed the effect of vortex development occurring under unsteady conditions. Due to the strong adverse pressure gradient in the deceleration phase of the cycle, vortex rings may detach at the proximal neck of the aneurysm, travel downstream, become unstable, and either decay or impinge on the distal wall. Nevertheless, in these studies, the wall shear stress was not measured.

In this context, a comprehensive analysis of the timevarying wall shear stress distribution is given in Peattie et al. (2004), where also the stability of the developing vortex rings are discussed. Salsac et al. (2006) also provides a thorough description of the unsteady wall shear stress, but does not consider the instability and laminar-turbulent transition of the vortex ring. Both studies from Peattie et al. (2004) and Salsac et al. (2006) investigate peak Reynolds numbers based on the undilated vessel diameter of $\mathrm{Re}=2300$ and $\mathrm{Re}=2700$, which is presumed to be too low for the human aorta (Kousera et al. 2013). Yip and Yu $(2001,2003)$ provide an overall description of the flow inside an aneurysm, and discuss the stability and decay of the vortex, as well as the spatial and temporal evolution of the WSS. Beside these experimental studies, there exist several numerical simulations of pulsating flow through generic aneurysms (Perktold 1987; Taylor and Yamaguchi 1994; Vorp et al. 1998; Finol et al. 2003), only to name a few.

The current work aims to contribute to both aforementioned approaches:

On the one hand, the pulsating flow through a generic aneurysm is analyzed in detail. The goal of this work is to provide a complete description of the flow phenomena in an aneurysm at transitional Reynolds numbers, and relate those phenomena to the spatial and temporal wall shear stress 
distribution. Special attention is drawn to the influence of the vortex ring on the wall shear stress.

On the other hand, this work is seen as a continuation of our former work (Bauer et al. 2019; Bopp et al. 2019), where the goal was to provide ground truth of the underlying WSS of acquired MRV data by comparison measurements and simulations. The MRV data acquired in the present work is intended to also be used as an input and reference for future WSS estimators. Many research groups struggle with this problem. However, an evaluation of different post-processing methods is only possible, if the exact same data set is used, and the underlying flow conditions and true WSS values are known. We therefore provide the acquired MRV data for other groups, which also may not have the possibility to conduct experiments, to test their WSS estimators. The present data set is available online (http://dx.doi.org/10.25534/ tudatalib-113.2) and can be regarded as a test case.

The experimental techniques used in this study are MRV and LDV. Besides the experimental measurements, numerical simulations are conducted with an improved instabilitysensitive Reynolds stress model (IISRSM), which is wellsuited for low Reynolds number flows and also for capturing laminar-turbulent transition and relaminarization.

The paper is structured as follows: First, the experimental setup and the numerical methods are described, followed by a detailed description of the flow phenomena present in the aneurysm by inspection of the MRV data. Comparison is drawn with LDV and CFD results. In a further comparison of LDV and MRV data, it is demonstrated that MRV image artifacts can be related to the occurrence of turbulence and that this information might be useful to identify laminar-turbulent transition. In a last step, the temporal and spatial distribution of the wall shear stress is examined, and discussed.

\section{Methods}

\subsection{Experimental setup}

As this work is a continuation of our former investigation (Bauer et al. 2019), most experimental procedures and setups remain similar. We will therefore only give a brief description here.

The experimental setup (Fig. 1a) consists of a portable flow supply unit, which can provide volume flow rates typical for the human aorta. The working fluid is water, doped with either a contrast agent or tracer particles, depending on the measurement technique. Experiments are performed at $22^{\circ} \mathrm{C}$, corresponding to a density of $\rho=997.75 \mathrm{~kg} / \mathrm{m}^{3}$ and a kinematic viscosity of $v=9.60 \times 7 \mathrm{~m}^{2} / \mathrm{s}$. The flow is guided with hoses to the measurement section in the MRI scanner room, before which several flow conditioning elements are inserted. Downstream of the flow conditioning, the fluid enters the aneurysm model, which is rigid, symmetrically, and smooth in shape, as shown in Fig. 1b. The maximum expansion of the diameter is $\alpha=D / d=2.5$, where $D$ is the maximum dilated diameter and $d$ the undilated pipe diameter of $d=26 \mathrm{~mm}$. The aneurysm has a length $L$ of $L / d=4$. The function for the aneurysm radius $r_{\mathrm{A}}$ in dependence of its dimensionless length $x / d \in[-2,2]$, where $x$ denotes the axial distance measured from the point of maximum diameter, is (a)

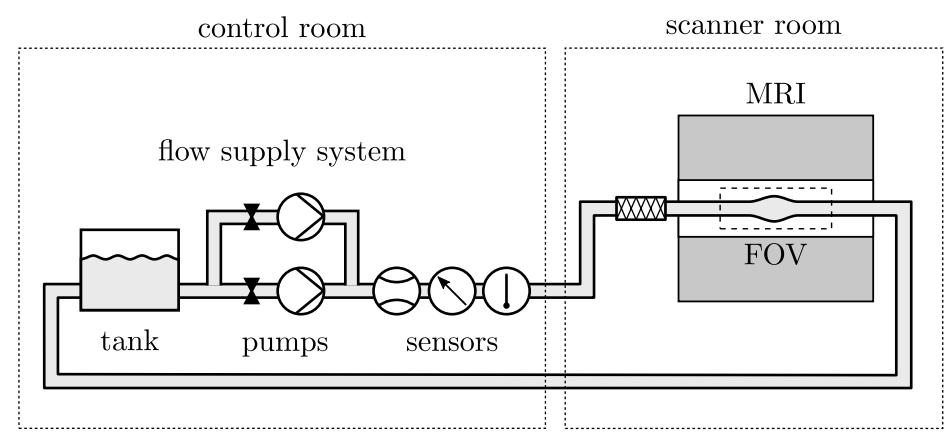

(b)

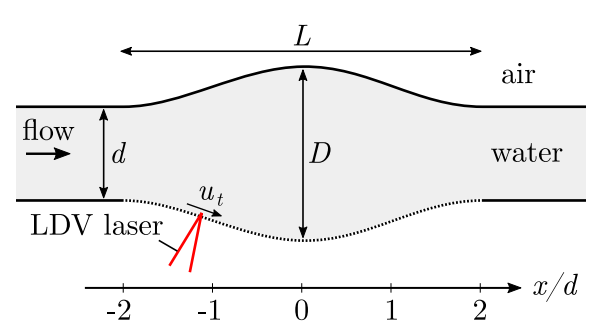

(c)

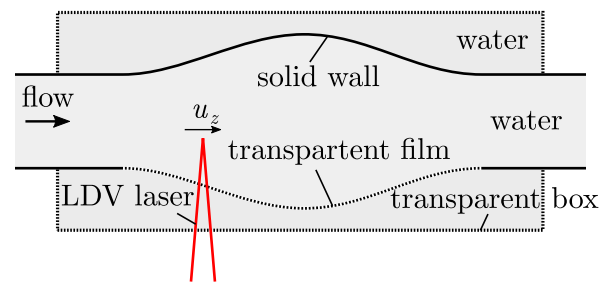

Fig. 1 a Experimental setup for MRV measurements. b Schematic representation of the aneurysm model including the geometric dimensions. This setup is used for measurements of the WSS, where the acquisition of wall-tangential velocities is possible. c Aneurysm model submerged in water for LDV measurements of axial velocities 


$$
\begin{aligned}
r_{\mathrm{A}}(x / d)= & \frac{1}{2}+\frac{\alpha-1}{2} \\
& \exp \left(4^{\gamma}-\left(0.25-\left(\frac{(x / d)+2}{4}-0.5\right)^{2}\right)^{-\gamma}\right),
\end{aligned}
$$

with $\gamma=0.7186$. The model is fabricated using a laser powder bed fusion process. To allow optical accessibility for the LDV measurements, the model has a thin slit, which is covered with a transparent film.

The temporal evolution of the flow is the same as in Bauer et al. (2019) for exercise conditions (case 3) and shown in Fig. 2. The flow waveform is adapted from Salsac et al. (2006) and resembles a physiological volume flow rate in the aorta. However, due to the large inertia of the moving fluid, the backflow and second peak around $t=2 \mathrm{~s}$ are obviously more pronounced than in in vivo flows. The peak Reynolds number based on the pipe diameter $d$ and the mean axial flow velocity $U$ is $\operatorname{Re}=U d / v=7649$. The Womersley number is Wo $=d / 2 \sqrt{2 \pi /(T v)}=20$, where $T$ is the cycle length of the pulsation, which corresponds to $T=2.7 \mathrm{~s}$.

Three-dimensional and time-resolved MRV measurements are performed at a $3 \mathrm{~T}$ Siemens Prisma whole body scanner, with receiving coils directly wrapped around the aneurysm. The MRV data were acquired with a k-t-accelerated 4D flow sequence (Bauer et al. 2013; Jung et al. 2008, 2011). After the MRV acquisition is completed, a so-called flow-off measurement is performed for which the pump is switched off. The flow model is not moved between those consecutive acquisitions. The flow-off data are used to retrospectively correct systematic background

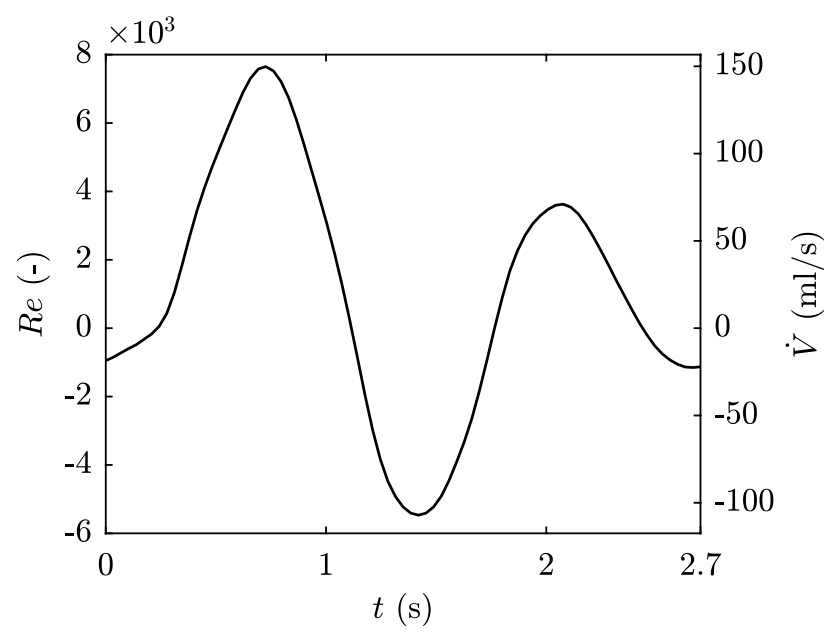

Fig. 2 Volume flow rate used in the present study, adapted from Bauer et al. (2019)
Table 1 MRV measurement parameters

\begin{tabular}{ll}
\hline Parameter & Value \\
\hline Sequence type & 3D3C, time resolved \\
Voxel size & $0.6 \mathrm{~mm}$ (isotropic) \\
venc & $0.25 \mathrm{~m} / \mathrm{s}$ \\
TE & $5.2 \mathrm{~ms}$ \\
TR & $128 \mathrm{~ms}$ \\
Phases & 21 \\
FOV & $216 \times 224 \times 224$ voxels \\
Acquisition time & $1: 39 \mathrm{~h}$ \\
Repetitions & 1 \\
\hline
\end{tabular}

phase errors, which might be induced, e.g., by eddy currents. Relevant MRV measurement parameters are shown in Table 1.

LDV measurements are carried out with a two-velocity component LDV system (Flow Explorer, Dantec Dynamics, wavelengths $\lambda_{1}=660 \mathrm{~nm}, \lambda_{2}=785 \mathrm{~nm}$, measurement volume size $331 \mu \mathrm{m} \times 49 \mu \mathrm{m} \times 49 \mu \mathrm{m}$ ).

For the measurement of velocity profiles, the aneurysm model is submerged in water (Fig. 1c). The refractive index of the transparent film is not equal to the refractive index of the surrounding fluid, but due to the thin thickness of only $0.5 \mathrm{~mm}$, this introduces just a minor shift of the measurement position. For the acquisition of the WSS, this setup does not allow an accurate determination of the wall-tangential velocity, since the radial velocity component cannot be measured. Therefore, the model is not submerged in water, but instead the LDV head is rotated for each measurement position so that the optical axis is perpendicular to the surface (Fig. 1b). The measurement of WSS requires only a penetration depth slightly deeper than the measurement volume size; thus, the curvature effect of the aneurysm wall is negligible.

Since the mean value of the axial velocity changes during the cycle, the calculation of the velocity fluctuation, respectively, RMS value would be biased. This problem has first been recognized by Hussain and Reynolds (1970), who proposed the so-called triple decomposition

$u=\bar{u}+\tilde{u}(\phi)+u^{\prime}$

where the velocity $u$ is decomposed into a time-averaged mean value $\bar{u}$, a phase-dependent part $\tilde{u}(\phi)$ and a fluctuating stochastic part $u^{\prime}$. In the present approach, the method proposed by Sonnenberger et al. (2000) is used to first obtain the phase-dependent part $\tilde{u}$. Therefore, a Fourier series up to the eighth order is fitted to the phase averaged LDV signal. Subsequently, the velocity fluctuations $u_{i}^{\prime}$ of each individual tracer particle can be calculated from 
$u_{i}^{\prime}=u_{i}-\bar{u}-\tilde{u}\left(\phi_{i}\right)$,

where $u_{i}$ is the measured particle velocity and $\phi_{i}$ its corresponding phase angle. In the circumferential velocity component, no mean flow is expected, thus $\bar{u}=\tilde{u}=0$ for this case. The mean and fluctuating velocity components are afterward binned into groups of the same bin width as the MRV data.

In addition to the measurements, an analytic solution is available for the inlet, assuming laminar, fully developed pulsating pipe flow (Womersley 1955; Durst et al. 1996; Bauer et al. 2019).

\subsection{Numerical methods}

The numerical simulations are conducted using the finite-volume-based open-source toolbox OpenFOAM ${ }^{\circledR}$. The URANS (Unsteady Reynolds-Averaged Navier-Stokes) model is used for the computation of this weakly turbulent flow in the aneurysm. The temporal discretization corresponds to the secondorder three-time step scheme with the Courant number adopted to be always smaller than 1 due to the use of controlled adaptive time steps. The central differencing scheme (CDS) is used for the discretization of the convective terms. The aneurysm geometry is meshed using OpenFOAMs build-in function 'blockMesh,' resulting in a three-dimensional hexahedral grid with $2.16 \times 10^{6}$ cells, which is shown in Fig. 3. At the inlet and outlet of the aneurysm, an additional length of $5 d$ is provided in each direction. The mesh is refined toward the wall, with the wall-next cells being located in the viscous sublayer at $y^{+}<1$ for the entire geometry and at all time steps.

Accordingly, an appropriately advanced turbulence model on the second-moment closure level is used. The near-wall Reynolds stress model by Jakirlić and Hanjalić (2002) is capable of separately capturing the kinematic wall blockage effects related to both Reynolds stress and stress dissipation anisotropies. The model is based on the homogeneous dissipation concept

$\varepsilon_{h}=\varepsilon-0.5 D_{k}^{v}$

with $\varepsilon$ representing the total viscous dissipation rate and $D_{k}^{v}$ the molecular diffusion of the kinetic energy of turbulence $k$. This satisfies inherently the exact asymptotic behavior of the stress dissipation and Reynolds stress components by approaching the solid wall, as well as resulting in a correct near-wall shape of the dissipation rate of the kinetic energy of turbulence without necessity to introduce any additional modifications. However, its straightforward temporal integration within the unsteady RANS method cannot adequately reproduce the turbulent activity intensification pertinent to a separated shear layer because of its time-averaged rationale. This is especially the case in flows which separate at a curved continuous wall, as encountered in the aneurysm configuration. Accordingly, this RANS model is further sensitized to account for fluctuating turbulence within the unsteady RANS framework, as proposed by Jakirlić and Maduta (2015). An additional term introduced into the corresponding length scale determining equation (governing the inverse time scale $\omega_{h}=\varepsilon_{h} / k$ ) provides a selective assessment of its production, which is in line with the scale-adaptive simulation (SAS) proposed by Menter and Egorov (2010). This enables the model capability to account for the vortex length and time scales variability related in particular to the highly unsteady separated shear layer regions. Presently, a somewhat different formulation of the additional production term is applied. Instead of the von Kármán length scale $L_{v K}$, as originally proposed by Menter and Egorov (2010), the term is modeled only in terms of the second velocity derivative, which originates from the equation governing the integral length scale (Rotta 1972). By doing so, the sensitivity of the model, which is denoted by improved instability-sensitive Reynolds stress model (IISRSM), in capturing the turbulence fluctuations is further enhanced. This enables the use of coarser grid resolutions.

\section{Velocity field}

Prior to the evaluation of the wall shear stress, the velocity field is examined more closely. First, the flow fields obtained with MRV and CFD are examined. It should be shown if the numerical simulation can capture the dynamics of the flow. Afterward, it is verified that LDV results agree well with MRV and CFD by comparing specific velocity profiles.
Fig. 3 Mesh of the computational domain including the aneurysm, inflow, outflow and precursor with the respective axial lengths (not true to scale)

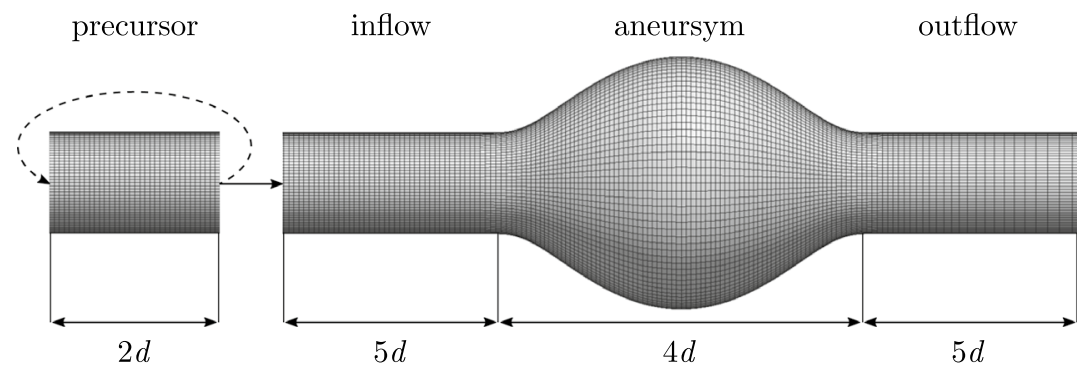




\subsection{Mean MRV velocity field}

The results of the MRV measurement are shown in a crosssectional (sagittal) view in Fig. 4. The CFD data of the same time steps are shown in Fig. 5 for comparison. Note that the MRV data are phase averaged, while the CFD data shown here correspond to an instant of time. Velocities are shown as velocity magnitudes in both cases.

At the beginning of the cycle $(t / T=0.07)$, the flow is close to zero and the velocity vectors are randomly oriented. As the flow rate increases $(t / T=0.21)$, a separation point develops at the inlet around $x / d \approx-1.8$, followed by a small recirculation zone downstream. The flow separation appears to have little influence on the velocity field downstream, where the flow is still fully attached. Subsequently, the flow rate reaches its maximum at $t / T=0.26$. At this time the pressure gradient is already negative, which enhances the flow separation at the proximal neck and the recirculation zone. A symmetrical vortex ring forms downstream of the point of detachment, with its center initially located at $x / d \approx-1.3$. At the wall, this vortex ring induces negative velocities. For the region $x / d>0$ , the flow is still fully attached and not influenced by the vortex. Subsequently, the flow rate rapidly decreases $(t / T=0.36)$, while the vortex ring reaches its maximum strength. Carried by the mean flow field, the vortex begins to travel downstream. In the middle of the aneurysm, surrounded by the vortex ring, a region with high velocities up to $u_{\mathrm{mag}}=0.33 \mathrm{~m} / \mathrm{s}$ exists. The vortex center induces reverse flow in the vicinity of the wall over a large area of $-1.0<x / d<0.2$. At $t / T=0.45$, the flow rate is close to its minimum value. At the wall, the flow is again fully attached over the entire aneurysm length, but with velocity vectors pointing in the upstream direction. At $t / T=0.55$ a secondary separation zone forms at the distal neck of the aneurysm at $x / d=1.5$. However, this region initially covers only a small region. Compared to the previous time step, the primary vortex ring has traveled upstream due to the negative flow rate and lost strength. Starting from $t / T=0.64$, the vortex ring begins to decay significantly. As the volume flow rate reaches its second maximum at $t / T=0.74$, the vortex is almost completely dissipated. In the region where the vortex impinged onto the wall, only a weak circulation and fluctuating velocities remain. At $t / T=0.83$, the flow rate approaches zero, and the flow in the aneurysm comes to a rest.

The overall agreement between CFD and MRV is very good.
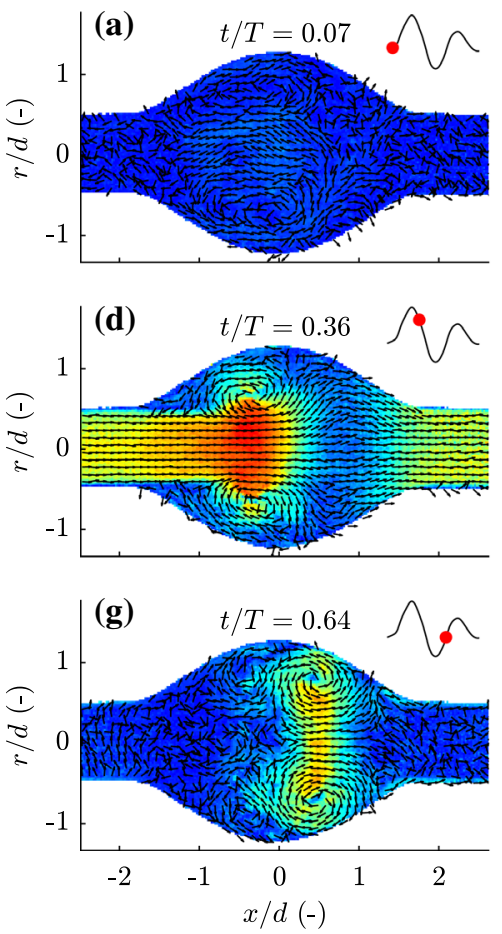
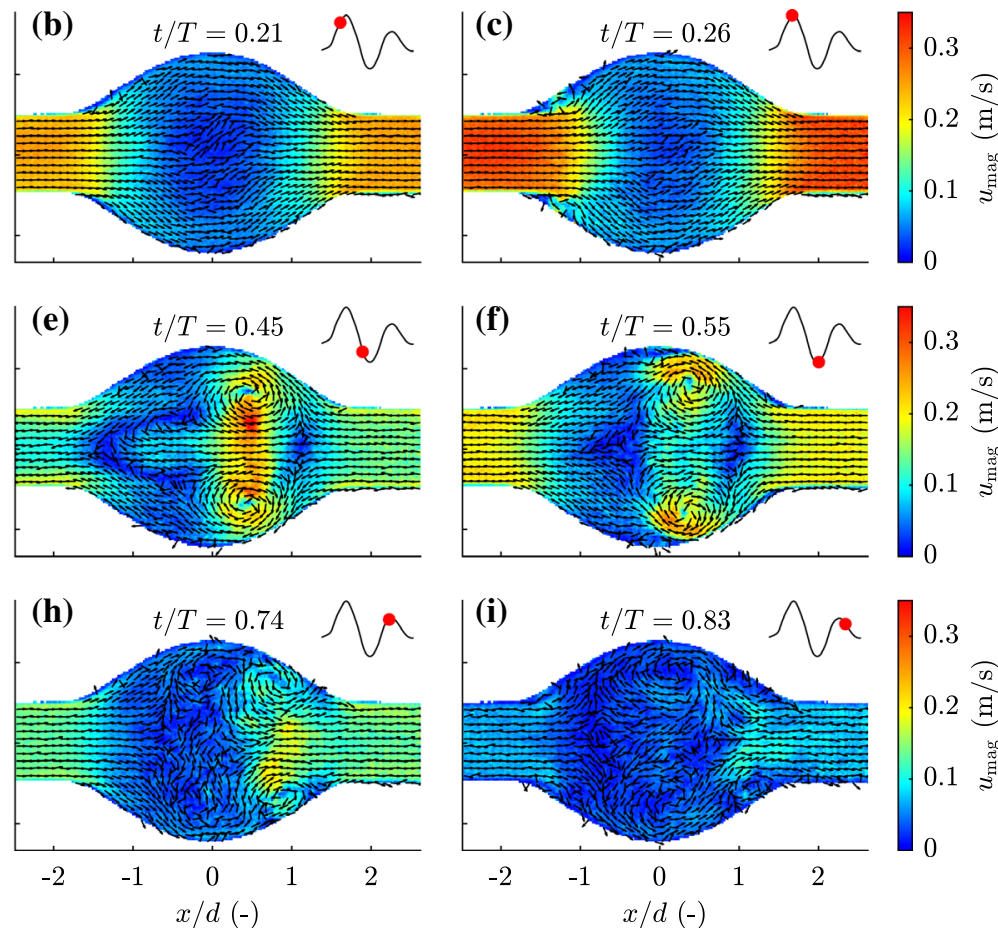
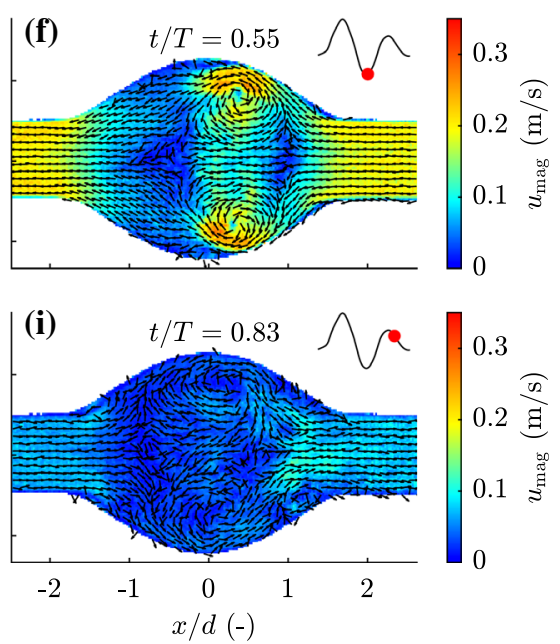

Fig. 4 Time series of the velocity field measured with MRV, in a cross-sectional (sagittal) view through the center of the aneurysm. At the beginning, the flow is fully attached (a), then the flow detaches at the proximal neck due to the increasing flow rate (b), and forms a vortex ring (c), which subsequently induces negative velocities at the wall, grows and travels downstream $(\mathbf{d}-\mathbf{e})$, weakens $(\mathbf{f}-\mathbf{g})$ and finally dissipates $(\mathbf{h}-\mathbf{i})$. Time steps within the cycle are visualized with the flow rate in the upper right corners 

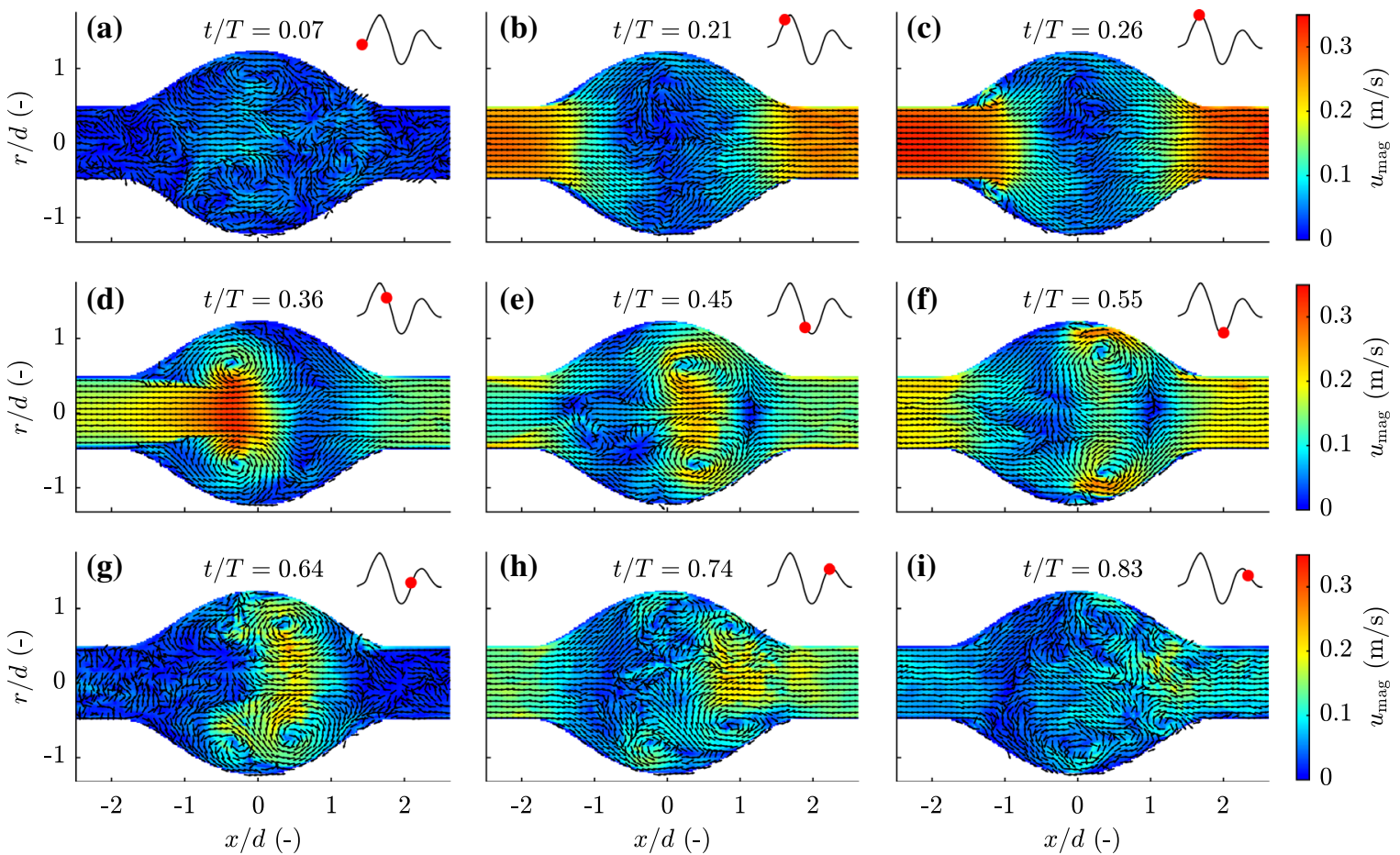

Fig. 5 Time series of the instantaneous velocity field from CFD, in a cross-sectional (sagittal) view through the center of the aneurysm. See Fig. 4 for further explanation

\subsection{Comparison with LDV data}

As suggested in other studies (Peattie et al. 2004; Yip and Yu 2001, 2003), the flow through an aneurysm may be in a laminar-turbulent transitional regime even though the upstream flow in the pipe is laminar. It is therefore necessary to verify whether the same flow conditions are present in both experimental setups and the simulation before proceeding further to the investigation of the WSS. The phased-averaged velocity profiles of the axial component from MRV, LDV and CFD are shown in Fig. 6. Since laser Doppler velocimetry is a point-by-point measurement technique, velocity profiles are acquired along straight lines between the center axis and the transparent wall at six different locations, which are at the inlet of the aneurysm $(x / d=-2.2)$, the point of separation $(x / d=-1.3)$, the center of the aneurysm $(x / d=0.0)$, the region where the vortex impinges and maximum WSS values are expected $(x / d=0.6$ and $x / d=1.0)$ and at the outlet $(x / d=2.2)$.

MRV velocity profiles are obtained by circumferential averaging. A detailed description of the procedure can be found in our first publication (Bauer et al. 2019). The flow field is divided into 12 equally spaced segments around the circumference, wherein the average velocity profile is calculated. The red curve in Fig. 6 represents the mean velocity over all segments, while the shaded area is the range, where all 12 velocity profiles fall. The shaded area is therefore a measure for flow symmetry. The CFD velocity profiles shown in Fig. 6 are averaged in circumferential direction similar to the MRV data. All profiles are evaluated at the peak volume flow rates of $t / T=0.26,0.55$ and 0.79 .

For $t / T=0.26$ (Fig. 6a) the mean values of MRV, LDV and CFD agree excellently. Flow separation starts to take place at the proximal neck $(x / d=-1.3)$, where the results already show negative velocities at the wall and a recirculation zone. Despite the periodic flow separation, the MRV velocity field shows no deviation from symmetry in the mean. The analytic laminar solution fits perfectly for the high volume flow rates at the inlet. Compared to the inlet, the velocity profile is significantly flattened at the aneurysm outlet. At the time of maximum negative volume flow rate $(t / T=0.55$, Fig. $6 \mathrm{~b})$, the vortex ring has reached the distal end of the aneurysm. Mean velocity profiles of MRV and LDV show again good agreement. The MRV data show an apparent asymmetric flow field. The numerical simulation reveals higher velocity amplitudes at $x / d=0$ and $x / d=0.6$ near the vortex. The analytic solution at the inlet does not fit the measurements well. For $t / T=0.79$ (Fig. 6c) slight deviations can be observed in the numerical simulation around $x / d=0.6$ and $x / d=1.0$.

Deviations of the mean MRV values from LDV near the center axis originate most likely from less voxels available at this position in the averaging process. Furthermore, MRV values at the wall are subjected to partial volume effects. In 

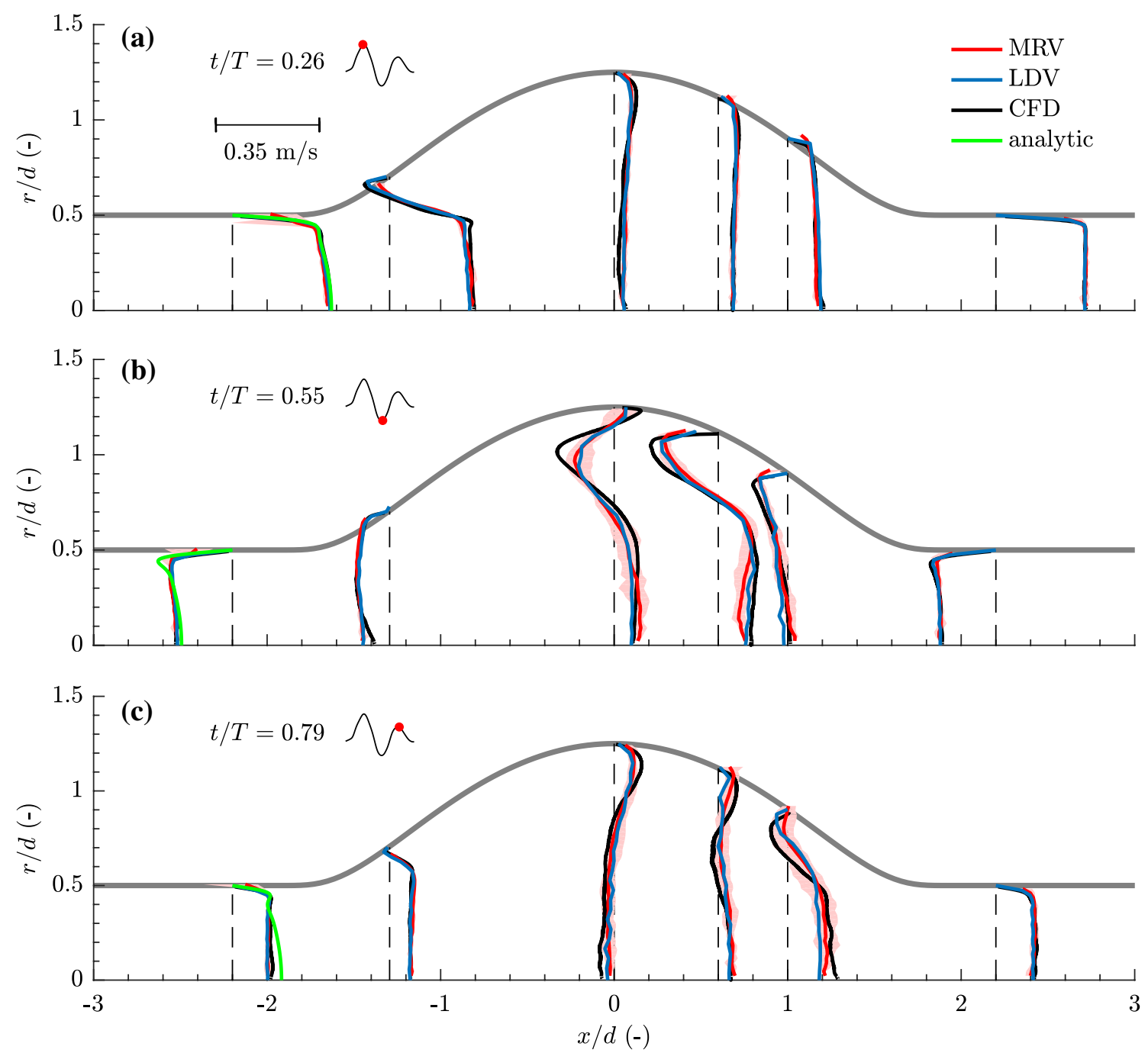

Fig. 6 Comparison of the axial velocity profiles at the three time steps of peak volume flow rate, acquired with MRV and LDV, compared to CFD and an analytic solution. The shaded red area indicates

both regions, the accuracy and reliability of the MRV data are therefore reduced. The results from LDV indicate that in the center of the aneurysm, the vortex introduces instabilities, as noticed in several other studies (Yip and Yu 2001, 2003; Peattie et al. 2004). It is therefore assumed that these instabilities lead to either weak turbulence or non-periodic velocity fluctuations between consecutive cycles. This might also cause the flattened velocity profile at the outlet in Fig. 6b compared to the inlet, due to enhanced mixing in the aneurysm bulk. WSS values are expected to be higher at the outlet than in the inlet. Inspection of the MRV velocities as well as the magnitude data shows that in the area, where the vortex ring introduces large velocity fluctuations, image artifacts occur, which potentially lead to the apparent deviations between individual MRV segments. The flow is therefore not necessarily asymmetric in these stages of the cycle, the variation of the MRV velocity profiles over the circumferential direction. In the first time step, a length scale for the velocity amplitudes is given

but affected by image artifacts due to velocity fluctuations. Deviations from the analytic solution at the inlet are caused by the back flow through the aneurysm; hence, not providing sufficient time to fully develop in the later phase of the cycle.

\subsection{Turbulence and velocity fluctuations}

In this section, the occurrence of turbulence is discussed.

MRV images are acquired by successively measuring the electromagnetic signal with different phase encoding gradients. As the acquisition takes a rather long time, the situation in the field of view (FOV) may be subject to changes, for instance caused by velocity fluctuations. Inconsistencies in the FOV during measurement will lead to inconsistencies between the k-space rows, assuming Cartesian sampling of the k-space. After transformation into image space using 
an inverse Fourier transformation, this will lead to image artifacts along the entire phase encoding direction. Random motion as well as random velocity fluctuations during image acquisition therefore results in an additional noise distribution, which are referred to as turbulence artifacts (Bruschewski et al. 2016).

Visual inspection of the MRV data suggests that this noise could be used as an indicator for laminar-turbulent transition. Figure 7 shows the MRV magnitude, where noise drastically increases during the time steps when the velocity fluctuations are present. The image artifacts appear around $x / d \approx 0$ and $t / T=0.55$ when the vortex ring introduces substantial velocity fluctuations. For a qualitative estimation of the laminar-turbulent transition, we now compare the timedependent velocity fluctuations obtained from LDV with the spatial image fluctuations and thus the velocity uncertainty from MRV.

In the LDV data, an axisymmetric turbulence $\left(u_{y}^{\prime}=u_{z}^{\prime}\right)$ is assumed and a mean fluctuation $u^{\prime}$ is computed

$u^{\prime}=\sqrt{\frac{1}{3}\left(u_{x}^{\prime 2}+2{u_{y}^{\prime 2}}^{2}\right)}$.

Additionally, a mean velocity fluctuation $\overline{u^{\prime}}$ is calculated for each of the six axial positions $x / d$ from Fig. 6 .

For the MRV data, the velocity uncertainty is calculated according to Constantinides et al. (1997) and Bruschewski et al. (2016) with $\sigma_{u, \mathrm{MRV}}=\frac{\mathrm{venc}}{\pi} \frac{1}{\mathrm{SNR}}$,

with the signal-to-noise ratio (SNR)

$\mathrm{SNR}=\frac{A}{\sigma}$.

In this formulation, $\sigma$ is the standard deviation of the magnitude in the background region and determined from

$\sigma=\sqrt{\left\langle\mathbf{M}^{2}\right\rangle / 2 L}$,

where $\mathbf{M}$ is the vector which contains the magnitude, \langle\rangle denotes arithmetic averaging and $L$ is the number of receiver coils. The noise-free magnitude value $A$ is calculated from the region of interest (ROI)

$A=\sqrt{\left\langle\mathbf{M}^{2}\right\rangle-2 L \sigma^{2}}$.

According to Bruschewski et al. (2016), the region in the background needs to be free of artifacts. However, as the intention of the present approach is to detect those artifacts, a region in the background along the phase encoding direction is used, which includes those artifacts.

Results of this method are shown in Fig. 8. Here, the velocity uncertainty from MRV is calculated over a slice located at $x / d=0.6$. Both methods show very good agreement. The uncertainty increases as the vortex ring approaches the slice and gradually decreases when the
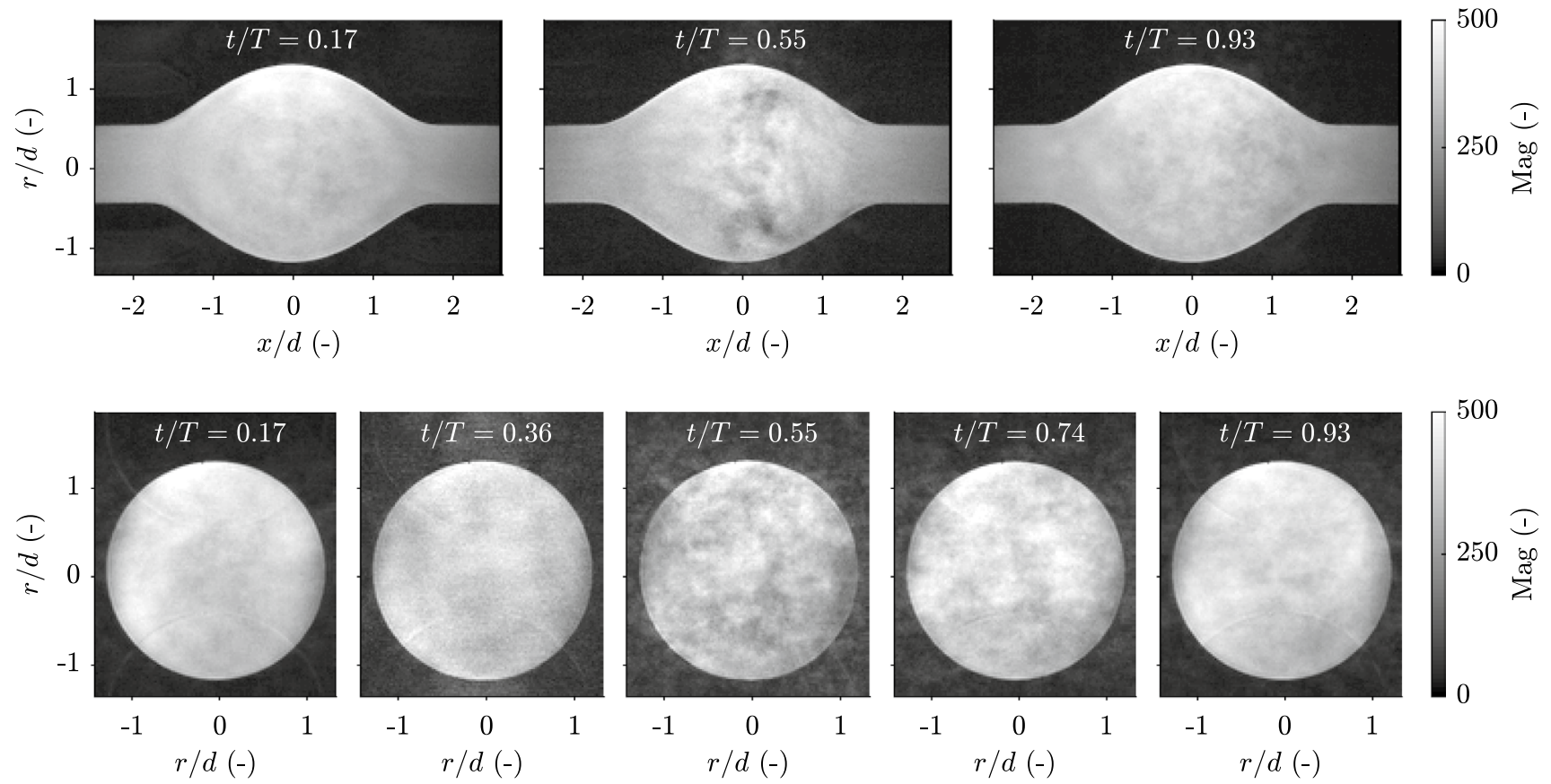

Fig. 7 Top row: MRV magnitude data in a sagittal plane in the middle of the aneurysm for three different time steps. The vortex decay introduces substantial image artifacts around $x / d \approx 0$ for $t / T=0.55$. Bottom row: Transverse plane at $x / d=0$ for five different time steps 


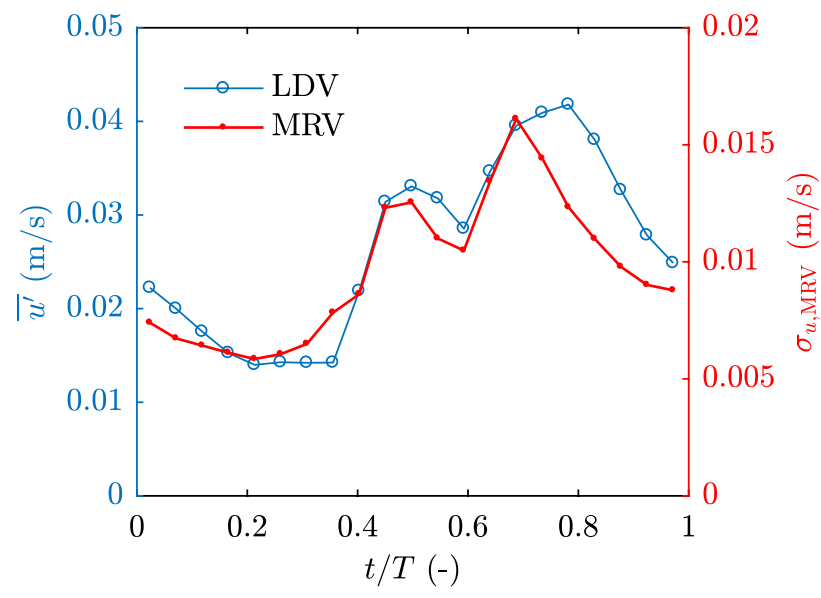

Fig. 8 Temporal evolution of the velocity fluctuations $\overline{u^{\prime}}$ from LDV and the velocity uncertainty $\sigma_{u \text { MRV }}$ from MRV in the slice located at $x / d=0.6$

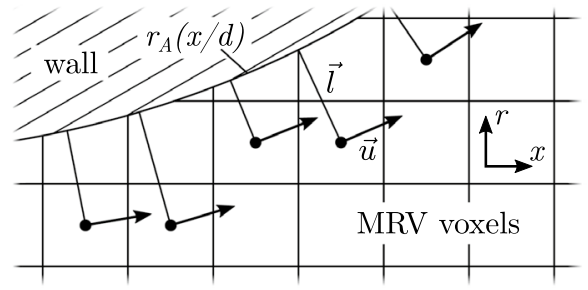

Fig. 9 For each axial slice in $x$-direction, the WSS is calculated between the first voxel not embedded into the wall and the wall with radius $r_{A}$

volume flow rate is negative and the vortex ring moves upstream $(t / T \approx 0.6)$; finally increasing again.

Thus, it is shown that laminar-turbulent transition appears in the LDV and MRV data at the same time, suggesting that the WSS values will be comparable. Overall, the agreement of the velocity fields between the experiments is also found to be excellent, so that a substantiated comparison of WSS values is now feasible.

\section{Wall shear stress}

The wall shear stress $\tau_{\mathrm{w}}$ from MRV is estimated from the circumferentially averaged velocity field, where the averaged data has the same spatial resolution as the original data. The velocity gradient is calculated for each axial slice between the first voxel completely inside the flow field and the wall position (Fig. 9), which is known from the wall function described in Eq. 1. At the wall, the no-slip condition is assumed. As previously shown in Bauer et al. (2019), this method is feasible; however, a symmetric flow field is required, which has been verified in Sect. 3.2.
For the determination of the wall position as well as the first measurement point totally inside the aneurysm with LDV, the procedure described in Bauer et al. (2019) is used in a steady flow. After that, the velocity gradient is measured with the unsteady volume flow rate. The LDV signal and thus the wall shear stress is phase averaged with four times the temporal resolution as MRV, which is at 84 time steps ( $\Delta t=32 \mathrm{~ms}$ intervals). The CFD data are obtained at 270 time steps ( $\Delta t=10 \mathrm{~ms}$ intervals).

The spatial distribution of the WSS from MRV, LDV and CFD for different time steps during the cycle is shown in Fig. 10. The circumferentially averaged velocity field from MRV is given on the bottom (using the right colorbar and the lower axis), while the WSS distribution of the corresponding time step is depicted on top (using the left axis). Additionally, the vortex centers of the flow field, are identified manually and their position is highlighted. The most prominent vortex ring is termed primary vortex, while all other vortices are referred to as secondary vortices.

In general, almost no deviation between the individual LDV measurement points and the numerical simulation is observed, while the results from MRV show significant underpredictions, especially in the regions and at the time steps where high WSS peaks are present. Below, the WSS distribution is discussed in detail for each time step.

While the volume flow rate is close to zero (Fig. 10a), the WSS is negligible small over the entire aneurysm length. In the middle of the flow field a secondary vortex is still present from the previous cycle, which does not influence the WSS.

When the flow rate increases (Fig. 10b), the global WSS distribution is almost similar to the distribution encountered in steady flows (Budwig et al. 1993; Bauer et al. 2019). The constant wall shear stress observed in the straight pipe drastically decreases at the separation point $(x / d=-1.6)$, followed by a small zone of weak recirculation and negative WSS. The wall shear stress then remains almost constant over the aneurysm length until the proximal end, where a distinct peak of $\tau_{\mathrm{w}}=0.85 \mathrm{~N} / \mathrm{m}^{2}$ is observed. Downstream of the aneurysm, the WSS continuously decreases due to the development of the laminar velocity profile in the pipe. In general, MRV is not capable to resolve the high WSS peaks at the distal and proximal ends, yielding an underestimation up to $56 \%$.

Due to the formation of the primary vortex from the recirculation zone (Fig. 10c), the results show a very narrow peak of $\tau_{\mathrm{w}}=-0.8 \mathrm{~N} / \mathrm{m}^{2}$ at $x / d \approx-1.3$. The strong vortex formation causes a secondary, minor and counter-rotating vortex to develop upstream, which cannot be resolved properly with the low spatial resolution of MRV, but is clearly visible from the CFD and WSS distribution. This leads to a localized positive WSS in the area around $x / d \approx-1.4$. At the outflow of the aneurysm, $\tau_{\mathrm{w}}$ reaches its maximum value during the entire cycle of $\tau_{\mathrm{w}}=1.1 \mathrm{~N} / \mathrm{m}^{2}$, which is again significantly 

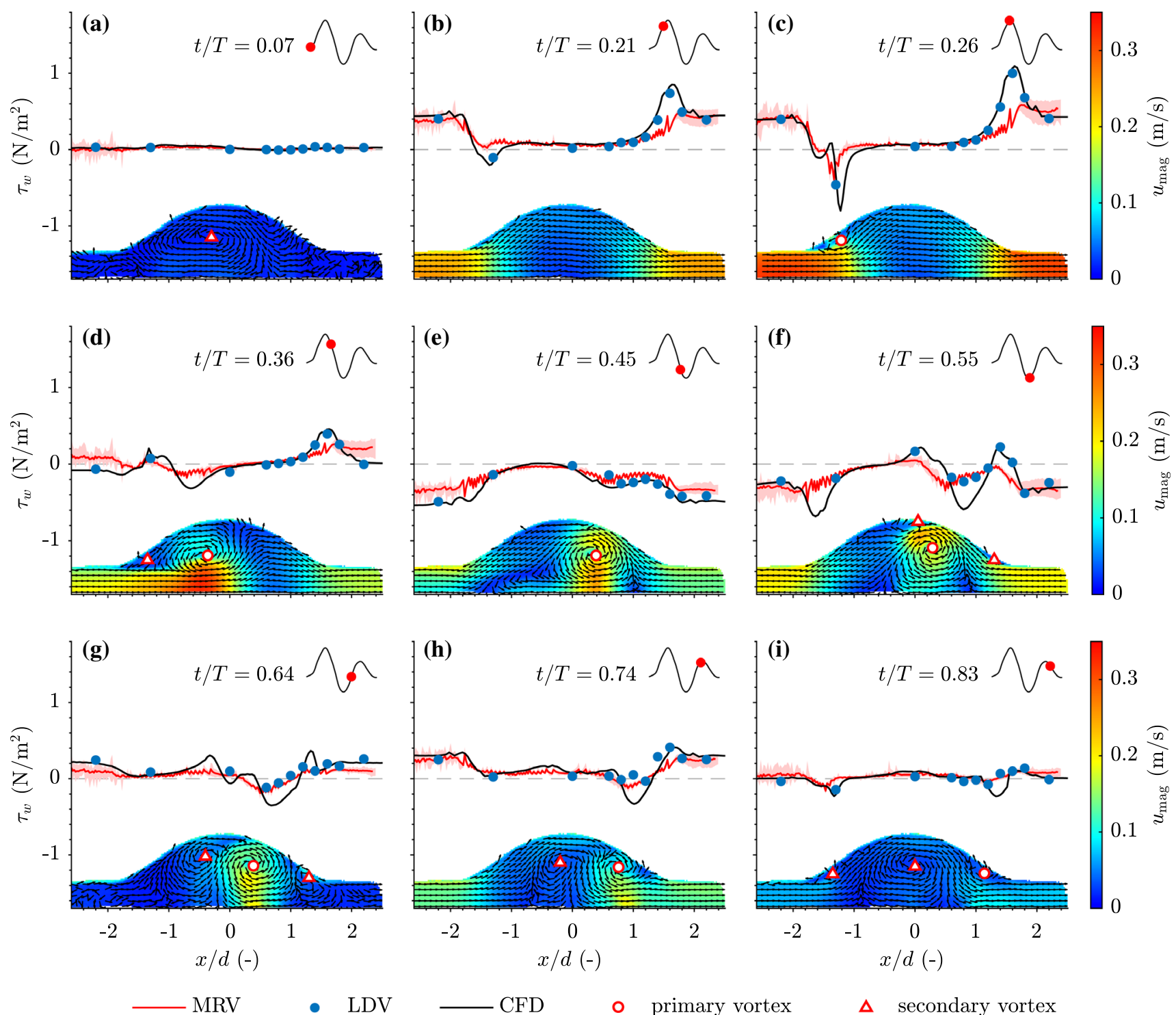

Fig. 10 Spatial distribution of the WSS for different time steps during the cycle. The respective flow rate at each time step is depicted in the upper right corners. The circumferentially averaged velocity field from MRV is given on the bottom (using the right colorbar and the

lower axis), while the spatial WSS distribution of the corresponding time step is depicted on top (using the left axis). WSS results from MRV, LDV and CFD are shown. Additionally, the vortex cores in the flow field are highlighted

underestimated by $54 \%$ with MRV. This systematic underestimation was already reported in (Bauer et al. 2019), where it occurred in steady flow conditions.

When the volume flow rate decreases, but is still positive (Fig. 10d), the pressure gradient is already negative. The velocities at the wall, where viscous forces dominate over inertia forces, may follow the pressure gradient faster than the flow in the middle of the cross section, causing a reversed flow at the wall and negative WSS in the straight pipes of the inlet and outlet. At this time step, results from MRV still predict a positive value, as the WSS from MRV is measured 1-2 voxels away from the wall; hence, those negative velocities cannot be detected with MRV. The influence of the primary and secondary vortices on the WSS is observed in the region $-1.5<x / d<0$. The primary vortex locally lowers the wall shear stress, while the secondary vortex leads to a positive WSS in its close proximity.

At $t / T=0.45$ (Fig. 10e), the secondary vortex is dissipated, and the primary vortex introduces only a minor additional WSS on the wall. Overall, at this time step no peaks of the WSS can be observed, and hence the MRV yields a reasonable estimate of the WSS, which is only slightly underestimated.

At the maximum negative volume flow rate (Fig. 10f), the primary vortex impinges onto the distal wall and induces high negative WSS values up to $\tau_{\mathrm{w}}=-0.6 \mathrm{~N} / \mathrm{m}^{2}$. 
In combination with the negative flow rate, the flow detaches from the wall just downstream of the primary vortex at $x / d=1.4$ and upstream in its wake around $x / d \approx 0$. Hence, two secondary vortices emerge. This phenomenon could not clearly been identified from the non-averaged velocity field in Fig. 4. At the proximal neck of the aneurysm, the identical phenomena are observed as for the forward flow discussed in Fig. 10b, Fig. 10c and the steady flows: The region where the cross section narrows is subjected to a peak in the WSS of $\tau_{\mathrm{w}}=-0.7 \mathrm{~N} / \mathrm{m}^{2}$. This time step represents the most complex flow situation in the cycle, since it contains three vortex rings and multiple stagnation and detachment points.

At $t / T=0.64$ (Fig. $10 \mathrm{~g}$ ), the flow situation is similar to the previous time step and highly complex with at least three vortices. The WSS calculation from MRV data completely fails to predict the WSS peaks here.

At the second maximum flow rate $(t / T=0.74$, Fig. $10 \mathrm{~h})$, the WSS distribution is comparable to the flow situation at $t / T=0.21$ (Fig. 10b), with a beginning detachment at the proximal end and a slightly increased WSS at the outflow. However, the primary vortex still introduces a WSS of $\tau_{\mathrm{w}}=-0.4 \mathrm{~N} / \mathrm{m}^{2}$ at the distal end.

In the last step $(t / T=0.83$, Fig. $10 \mathrm{i})$, the overall wall shear stress is close to zero, except at the proximal $(x / d=-1.5)$ and distal $(x / d=1.3)$ ends, where the primary and a secondary vortices lead to $\tau_{\mathrm{w}} \approx-0.1 \mathrm{~N} / \mathrm{m}^{2}$.

\section{Discussion}

In summary, a vortex originates at every peak of the volume flow rate at the location, where the cross section expands, which is the proximal end for positive flow rates and the distal end for negative flow rate. The wall shear stress is significantly altered in the region where those vortices are located in close proximity to the wall. The most prominent WSS peaks are found at the outlet of the aneurysm when the vortex impinges onto the wall, at the distal end at the highest volume flow rate, and at the position where the primary vortex initially develops. Compared to the first two peaks, the latter peak is restricted not only to a very narrow spatial region of $\Delta x / d \approx 0.2$, but also to a short time interval. It is worth noting that the temporal gradient of the wall shear stress is most prominent at that position. Although the absolute values are higher at the distal end, their temporal evolution is somewhat predictable and the distribution changes only slowly. Compare for example the evolution of the high WSS value at $x / d=1.6$ between Fig. $10 \mathrm{~b}$ and $10 \mathrm{c}$, or the high negative value at the same location between Fig. $10 \mathrm{f}$ and $\mathrm{g}$ with the temporal change at $x / d=-1.3$ between Fig. 10c and 10d.

For a more substantiated and quantitative evaluation of the temporal change, it is reasonable to calculate the temporal wall shear stress gradient (TWSSG), which is a measure of the temporal change of the WSS at the respective spatial location. This quantity has also been discussed in the literature, where the endothelium was found to be sensitive to the TWSSG (White et al. 2001). The TWSSG is calculated from a central differencing scheme according to

$\operatorname{TWSSG}=\left.\frac{\partial \tau_{\mathrm{w}}(x, t)}{\partial t}\right|_{t=t_{i}}=\frac{\tau_{\mathrm{w}}\left(t_{i}+\Delta t\right)-\tau_{\mathrm{w}}\left(t_{i}-\Delta t\right)}{2 \Delta t}$,

where $t_{i}$ is the respective time step in the cycle and $\Delta t$ the step size between two consecutive time steps. Results of the calculation of the TWSSG are shown in Fig. 11. Overall, the magnitude of TWSSG is below $\pm 4 \mathrm{~N} /\left(\mathrm{m}^{2} \mathrm{~s}\right)$ for the entire cycle, except at $t / T=0.26$ (Fig. 11c). At this point, an exceptionally high peak of TWSSG $= \pm 10 \mathrm{~N} /\left(\mathrm{m}^{2} \mathrm{~s}\right)$ is located at the position where the high temporal change from the vortex development was initially assumed. In agreement with the CFD, the results from LDV show the same peak. Hence, the temporal evolution of the WSS at the point of formation of the primary vortex $(x / d=-1.3)$ is now analyzed in more detail.

The WSS at $x / d=-1.3$ is shown in Fig. 12. At the time, the vortex ring starts to develop $(t / T \approx 0.2)$, the WSS rapidly decreases from $\tau_{\mathrm{w}}=0 \mathrm{~N} / \mathrm{m}^{2}$ to $\tau_{\mathrm{w}}=-0.55 \mathrm{~N} / \mathrm{m}^{2}$ and subsequently increases again to $\tau_{\mathrm{w}}=0.17 \mathrm{~N} / \mathrm{m}^{2}$. While CFD and LDV results agree excellently up to $t / T=0.3$ and good in the later cycle, the MRV results systematically underestimate the WSS, especially the negative peak at $t / T=0.2$. This peak only appears in a relatively short time interval of $\Delta t / T \approx 0.1$, while the temporal resolution of the MRV measurement is only $\Delta t / T \approx 0.05$. Thus, it is assumed that the underprediction from MRV not only originates from the low spatial resolution, but also from the limited temporal resolution.

To demonstrate the effect of the intrinsic temporal averaging of MRV, the temporally highly resolved WSS results from CFD and the phase-locked signal from LDV, both located at $x / d=-1.3$, are phase averaged with different numbers of time steps during the cycle. The minimum number of time steps is 1 , corresponding to an overall mean value. Using 21 time steps yields the same temporal resolution as the MRV measurement. In Fig. 13, the underestimation of the peak at $t / T=0.2$ due to the lower temporal resolution is shown in relation to the number of time steps.

As expected, the use of fewer time steps yields a more significant underestimation. The value measured with MRV is also depicted in Fig. 13. The underestimation is further enhanced due to the limited spatial resolution. A high scatter can be noticed, which can be explained with the location of the time steps relative to the minimum of the WSS peak. The upper bound of the distribution represents the situation when a time step coincides with the position of the peak, yielding 

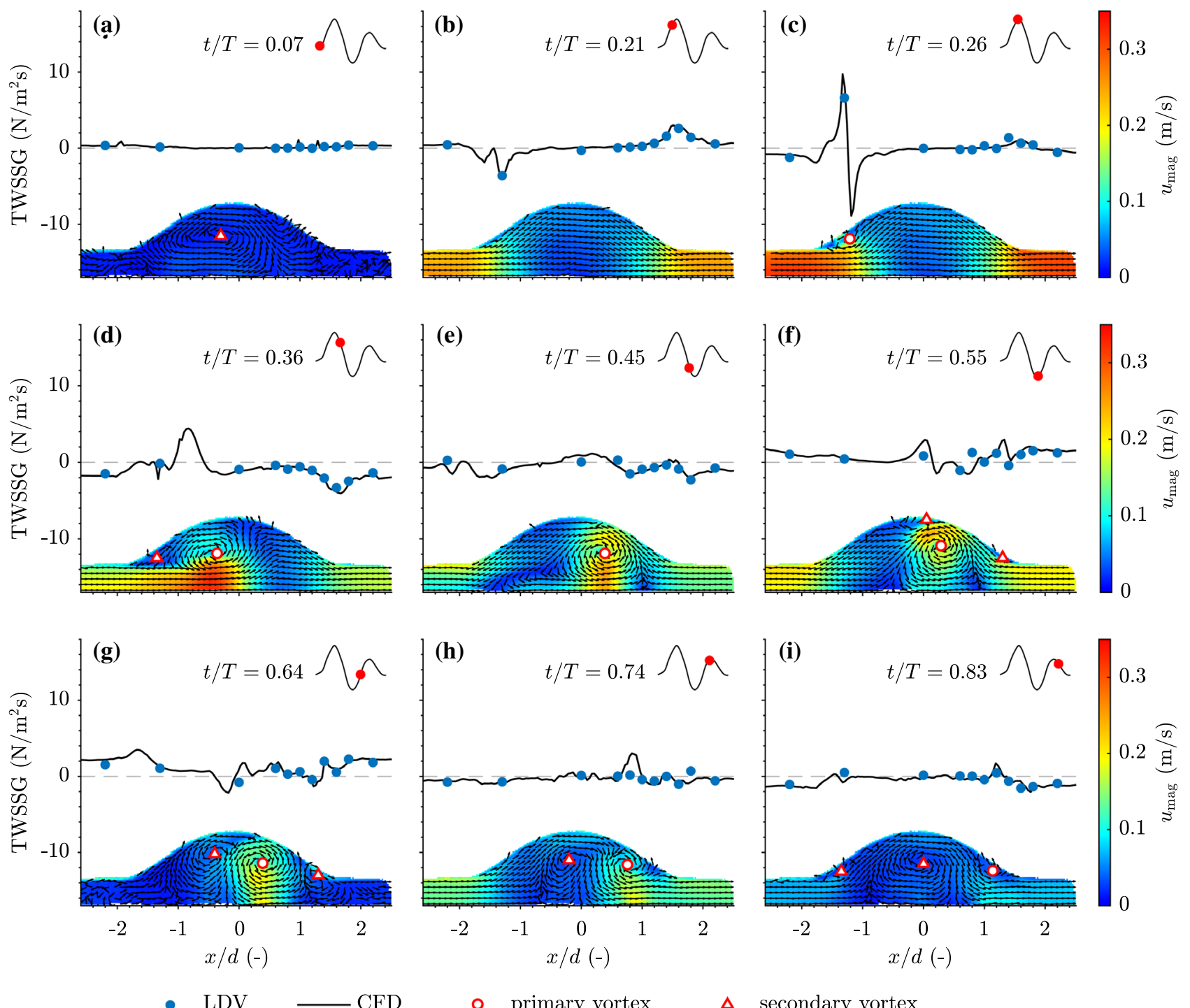

- LDV $\longrightarrow$ CFD

○ primary vortex

$\Delta \quad$ secondary vortex

Fig. 11 Temporal wall shear stress gradient (TWSSG) for different time steps during the cycle. A distinct peak is observed when the primary vortex detaches at the proximal end $(t / T=0.26)$. The circumferentially averaged velocity field from MRV is given on the bottom

less underestimation, while the lower bound represents a position of the time steps relative to the peak resulting in a pronounced underestimation.

\section{Conclusion}

The experimental results presented in this work show good agreement regarding the velocity field. The laminar-turbulent transition could be detected and reproduced in both experimental investigations and modeled in the simulation. In accordance with previous investigations, a strong vortex ring was observed in the aneurysm, which substantially (using the right colorbar and the lower axis), while the TWSSG distribution of the corresponding time step is depicted on top (using the left axis). Results from LDV and CFD are shown. Additionally, the vortex cores in the flow field are highlighted

influenced the WSS distribution and lead to high wall shear stress amplitudes when it is located close to the wall.

It has to be emphasized that the methods used in this work for the estimation of the WSS from MRV are rather simple. There exist far more advanced methods in the literature, which may yield better results. Nevertheless, it was not the purpose of this work to develop new WSS estimators, but to reveal the 'true' value of the WSS distribution for a flow acquired with MRV.

The agreement of the wall shear stress between LDV and CFD was found to be excellent. Thus, the numerical simulation can be regarded as the 'ground truth' value of the WSS. In a next step, different estimators and postprocessing methods can be applied to the MRV data, and 


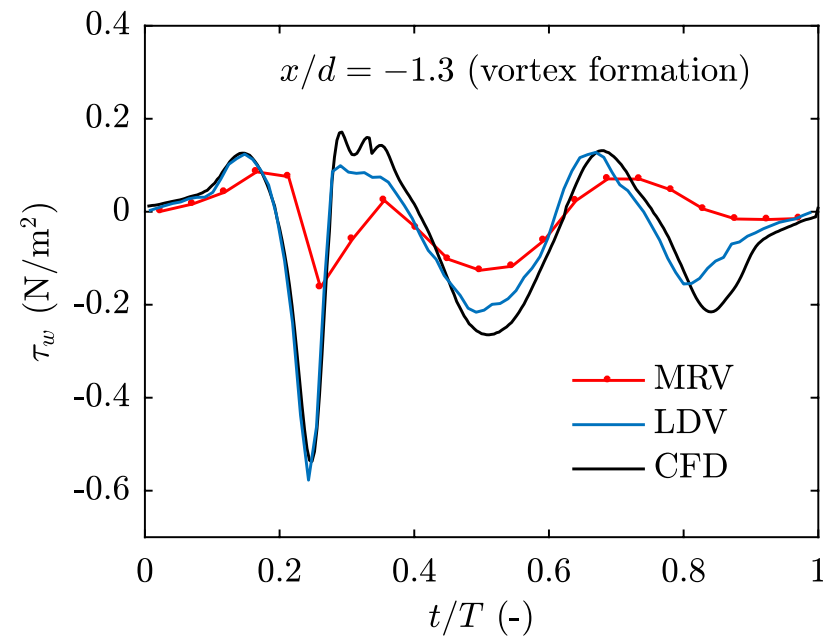

Fig. 12 Temporal evolution of the WSS at the position where the primary vortex initially develops $(x / d=-1.3)$. The vortex development close to the wall leads to a high negative WSS for a very short time period $(\Delta t / T=0.1)$, which cannot be resolved properly with MRV

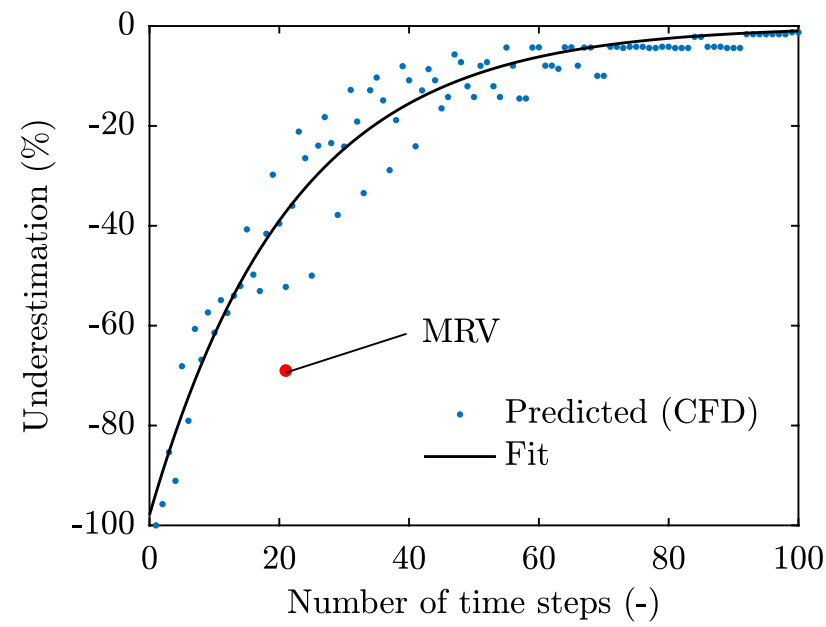

Fig. 13 Theoretical underestimation of the wall shear stress at $x / d=-1.3$ in dependence on the number of time steps. The measured value with MRV may be further underestimated due to the low spatial resolution

the results can be compared to the numerical simulations and LDV values of this work. As already stated in the introduction, the present MRV data are available online (http://dx.doi.org/10.25534/tudatalib-113.2) and can be used by other authors to test their own WSS estimators.

Furthermore, it was shown that the measurement uncertainty of the MRV data can be used to yield information about the flow state. However, it is not possible to quantify absolute values of the velocity fluctuations, but rather estimate the relative increase in fluctuations. Since this method does not require additional measurement time, it seems to be a valuable secondary information.

It is well-known that the spatial resolution of MRV is not sufficient to accurately determine the wall shear stress directly from the near-wall velocity gradient. However, it is in general not assumed that a low temporal resolution can reduce the wall shear stress estimation in the same order as the spatial resolution does (Montalba et al. 2018; Zimmermann et al. 2018). With the use of the temporally highresolved LDV and CFD data of this work, it was shown that the underprediction may indeed be very large. Even for the case of a sufficiently high spatial resolution, an underprediction of approximately $40 \%$ is expected with the present number of phases during the cycle. Nevertheless, this effect is only present at the location where the primary vortex ring develops. At the locations of the secondary minor vortices, this effect has not been observed. This indicates that for an accurate determination of the wall shear stress, it is not sufficient to calculate the WSS from individual MRV images in a step-by-step procedure without a proper representation of the flow field in-between those time steps. It is furthermore assumed that this effect cannot be solved by an interpolation between consecutive time steps. As a solution for abdominal aneurysms where strong vortices appear, it is reasonable to use dynamic MRI sequences with a high temporal resolution.

Acknowledgements Open Access funding provided by Projekt DEAL. The authors would like to thank the German Research Foundation (DFG) for financially supporting this project under the Grants TR 194/56-1 and HE 1875/30-1.

Open Access This article is licensed under a Creative Commons Attribution 4.0 International License, which permits use, sharing, adaptation, distribution and reproduction in any medium or format, as long as you give appropriate credit to the original author(s) and the source, provide a link to the Creative Commons licence, and indicate if changes were made. The images or other third party material in this article are included in the article's Creative Commons licence, unless indicated otherwise in a credit line to the material. If material is not included in the article's Creative Commons licence and your intended use is not permitted by statutory regulation or exceeds the permitted use, you will need to obtain permission directly from the copyright holder. To view a copy of this licence, visit http://creativecommons.org/licenses/by/4.0/.

\section{References}

Aggarwal S, Qamar A, Sharma V, Sharma A (2011) Abdominal aortic aneurysm: a comprehensive review. Exp Clin Cardiol 16(1):11

Arzani A, Shadden SC (2016) Characterizations and correlations of wall shear stress in aneurysmal flow. J Biomed Eng 138(1):014503

Asbury CL, Ruberti JW, Bluth EI, Peattie RA (1995) Experimental investigation of steady flow in rigid models of abdominal aortic aneurysms. Ann Biomed Eng 23(1):29-39

Bauer A, Wegt S, Bopp M, Jakirlic S, Tropea C, Krafft AJ, Shokina N, Hennig J, Teschner G, Egger H (2019) Comparison of wall 
shear stress estimates obtained by laser Doppler velocimetry, magnetic resonance imaging and numerical simulations. Exp Fluids 60(7): 112

Bauer S, Markl M, Föll D, Russe M, Stankovic Z, Jung B (2013) K-t GRAPPA accelerated phase contrast MRI: improved assessment of blood flow and 3-directional myocardial motion during breathhold. J Magn Reson Imaging 38(5):1054-1062

Bopp M, Bauer A, Wegt S, Jakirlic S, Tropea C (2019) A computational and experimental study of physiological pulsatile flow in an aortic aneurysm. In: 11th International symposium on turbulence and shear flow phenomena, Southampton, UK

Boussel L, Rayz V, McCulloch C, Martin A, Acevedo-Bolton G, Lawton M, Higashida R, Smith WS, Young WL, Saloner D (2008) Aneurysm growth occurs at region of low wall shear stress: patient-specific correlation of hemodynamics and growth in a longitudinal study. Stroke 39(11):2997-3002

Boyd AJ, Kuhn DC, Lozowy RJ, Kulbisky GP (2016) Low wall shear stress predominates at sites of abdominal aortic aneurysm rupture. J Vasc Surg 63(6):1613-1619

Bruschewski M, Freudenhammer D, Buchenberg W, Schiffer H-P, Grundmann S (2016) Estimation of the measurement uncertainty in magnetic resonance velocimetry based on statistical models. Exp Fluids 57(5):57-83

Budwig R, Elger D, Hooper H, Slippy J (1993) Steady flow in abdominal aortic aneurysm models. J Biomed Eng 115(4A):418-423

Constantinides CD, Atalar E, McVeigh ER (1997) Signal-to-noise measurements in magnitude images from NMR phased arrays. Magn Reson Med 38(5):852-857

Deplano V, Guivier-Curien C, Bertrand E (2016) 3D analysis of vortical structures in an abdominal aortic aneurysm by stereoscopic PIV. Exp Fluids 57(11):167

Deplano V, Knapp Y, Bertrand E, Gaillard E (2007) Flow behaviour in an asymmetric compliant experimental model for abdominal aortic aneurysm. J Biomech 40(11):2406-2413

Deplano V, Meyer C, Guivier-Curien C, Bertrand E (2013) New insights into the understanding of flow dynamics in an in vitro model for abdominal aortic aneurysms. Med Eng Phys 35(6):800-809

Dolan JM, Kolega J, Meng H (2013) High wall shear stress and spatial gradients in vascular pathology: a review. Ann Biomed Eng 41(7):1411-1427

Durst F, Ismailov M, Trimis D (1996) Measurement of instantaneous flow rates in periodically operating injection systems. Exp Fluids 20:178-188

Egelhoff C, Budwig R, Elger D, Khraishi T, Johansen K (1999) Model studies of the flow in abdominal aortic aneurysms during resting and exercise conditions. J Biomech 32(12):1319-1329

Fillinger MF, Raghavan ML, Marra SP, Cronenwett JL, Kennedy FE (2002) In vivo analysis of mechanical wall stress and abdominal aortic aneurysm rupture risk. J Vasc Surg 36(3):589-597

Finol E, Keyhani K, Amon C (2003) The effect of asymmetry in abdominal aortic aneurysms under physiologically realistic pulsatile flow conditions. J Biomed Eng 125(2):207-217

Hussain AKMF, Reynolds WC (1970) The mechanics of an organized wave in turbulent shear flow. J Fluid Mech 41(2):241-258

Jakirlić S, Hanjalić K (2002) A new approach to modelling nearwall turbulence energy and stress dissipation. J Fluid Mech 459:139-166

Jakirlić S, Maduta R (2015) Extending the bounds of 'steady' RANS closures: toward an instability-sensitive Reynolds stress model. Int J Heat Fluid Flow 51:175-194

Jung B, Stalder AF, Bauer S, Markl M (2011) On the undersampling strategies to accelerate time-resolved 3D imaging using k-tGRAPPA. Magn Reson Med 66(4):966-975

Jung B, Ullmann P, Honal M, Bauer S, Hennig J, Markl M (2008) Parallel MRI with extended and averaged GRAPPA kernels
(PEAK-GRAPPA): optimized spatiotemporal dynamic imaging. J Magn Reson Imaging 28(5):1226-1232

Kemmerling EM, Peattie RA (2018) Abdominal aortic aneurysm pathomechanics: current understanding and future directions. In: Fu $\mathrm{BM}$, Wright NT (eds) Molecular, cellular, and tissue engineering of the vascular system. Springer, Berlin, pp 157-179

Kousera C, Wood N, Seed W, Torii R, O'regan D, Xu X (2013) A numerical study of aortic flow stability and comparison with in vivo flow measurements. J Biomed Eng 135(1):011003

Lasheras JC (2007) The biomechanics of arterial aneurysms. Annu Rev Fluid Mech 39:293-319

Markl M, Frydrychowicz A, Kozerke S, Hope M, Wieben O (2012) 4D flow MRI. J Magn Reson Imaging 36(5):1015-1036

Meng H, Wang Z, Hoi Y, Gao L, Metaxa E, Swartz DD, Kolega J (2007) Complex hemodynamics at the apex of an arterial bifurcation induces vascular remodeling resembling cerebral aneurysm initiation. Stroke 38(6):1924-1931

Menter FR, Egorov Y (2010) The scale-adaptive simulation method for unsteady turbulent flow predictions. Part 1: theory and model description. Flow Turbul Combust 85(1):113-138

Miura Y, Ishida F, Umeda Y, Tanemura H, Suzuki H, Matsushima S, Shimosaka S, Taki W (2013) Low wall shear stress is independently associated with the rupture status of middle cerebral artery aneurysms. Stroke 44(2):519-521

Montalba C, Urbina J, Sotelo J, Andia ME, Tejos C, Irarrazaval P, Hurtado DE, Valverde I, Uribe S (2018) Variability of 4D flow parameters when subjected to changes in MRI acquisition parameters using a realistic thoracic aortic phantom. Magn Reson Med 79(4):1882-1892

Munarriz PM, Gómez PA, Paredes I, Castaño-Leon AM, Cepeda S, Lagares A (2016) Basic principles of hemodynamics and cerebral aneurysms. World Neurosurg 88:311-319

Peattie RA, Riehle TJ, Bluth EI (2004) Pulsatile flow in fusiform models of abdominal aortic aneurysms: flow fields, velocity patterns and flow-induced wall stresses. J Biomed Eng 126(4):438-446

Perktold K (1987) On the paths of fluid particles in an axisymmetrical aneurysm. J Biomech 20(3):311-317

Rotta JC (1972) Turbulente Strömungen: Eine Einführung in die Theorie und ihre Anwendung. Teubner Verlag, Stuttgart

Salsac A-V, Sparks S, Chomaz J-M, Lasheras J (2006) Evolution of the wall shear stresses during the progressive enlargement of symmetric abdominal aortic aneurysms. J Fluid Mech 560:19-51

Sonnenberger R, Graichen K, Erk P (2000) Fourier averaging: a phaseaveraging method for periodic flow. Exp Fluids 28(3):217-224

Taylor TW, Yamaguchi T (1994) Three-dimensional simulation of blood flow in an abdominal aortic aneurysm - steady and unsteady flow cases. J Biomech Eng Trans ASME 116(1):89-97

van Ooij P, Markl M, Collins JD, Carr JC, Rigsby C, Bonow RO, Malaisrie SC, McCarthy PM, Fedak PW, Barker AJ (2017) Aortic valve stenosis alters expression of regional aortic wall shear stress: new insights from a 4-dimensional flow magnetic resonance imaging study of 571 subjects. J Am Heart Assoc 6(9):e005959

Vorp DA, Raghavan M, Webster MW (1998) Mechanical wall stress in abdominal aortic aneurysm: influence of diameter and asymmetry. J Vasc Surg 27(4):632-639

Watton P, Selimovic A, Raberger NB, Huang P, Holzapfel G, Ventikos Y (2011) Modelling evolution and the evolving mechanical environment of saccular cerebral aneurysms. Biomech Model Mech 10(1):109-132

White CR, Haidekker M, Bao X, Frangos JA (2001) Temporal gradients in shear, but not spatial gradients, stimulate endothelial cell proliferation. Circulation 103(20):2508-2513

Womersley JR (1955) Method for the calculation of velocity, rate of flow and viscous drag in arteries when the pressure gradient is known. J Physiol Lond 127:553-563 
Yip T, Yu S (2001) Cyclic transition to turbulence in rigid abdominal aortic aneurysm models. Fluid Dyn Res 29(2):81-113

Yip T, Yu S (2003) Cyclic flow characteristics in an idealized asymmetric abdominal aortic aneurysm model. Proc Inst Mech Eng H 217(1):27-39

Zimmermann J, Demedts D, Mirzaee H, Ewert P, Stern H, Meierhofer C, Menze B, Hennemuth A (2018) Wall shear stress estimation in the aorta: impact of wall motion, spatiotemporal resolution, and phase noise. J Magn Reson Imaging 48(3):718-728
Publisher's Note Springer Nature remains neutral with regard to jurisdictional claims in published maps and institutional affiliations.

\section{Affiliations}

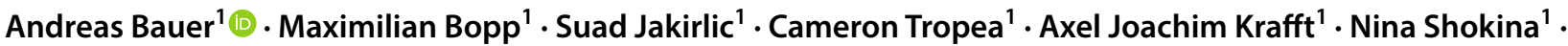 Jürgen Hennig ${ }^{1}$}

1 Institute of Fluid Mechanics and Aerodynamics, Technische Universität Darmstadt, Darmstadt, Germany 